\title{
Yang-Mills-Higgs Monopole Solutions of Arbitrary Topological Charge
}

\author{
M. K. Prasad \\ Department of Mathematics, Massachusetts Institute of Technology, Cambridge, MA 02139, USA
}

\begin{abstract}
We propose a construction of static magnetic Yang-Mills-Higgs monopole solutions of arbitrary topological charge. They are axially symmetric and contain no free parameters except for their position. The regularity of the solutions has yet be proved; doing so would complete the constructive proof of existence.
\end{abstract}

\section{Introduction}

The purpose of this paper is to present new static magnetic Yang-Mills-Higgs monopole solutions of arbitrary topological charge. The solutions are axially symmetric and contain no free parameters except for their position in three dimensional Euclidean space. Our solution thus generalizes the recent and remarkable construction of Ward [1] on the charge 2 monopole. Unlike Ward's construction, our construction is based on a systematic framework for obtaining monopole solutions with arbitrary topological charge. As a byproduct of the systematic framework, we are able to explicitly construct the complex gauge transformation that makes Ward's (and our) solution real. In a separate paper [9] we have verified regularity of these solutions in various regions, but we have not yet shown that these regions cover the three dimensional Euclidean space.

Let us define in four dimensional Euclidean space $\left(x_{1}, x_{2}, x_{3}, x_{4}\right)$ the gauge potentials $A_{\mu}^{a}$ where $a=1,2,3$ and $\mu=1,2,3,4$. The gauge field strength is defined by

$$
F_{\mu \nu}^{a} \equiv \partial_{\mu} A_{v}^{a}-\partial_{\nu} A_{\mu}^{a}+e \varepsilon^{a b c} A_{\mu}^{b} A_{v}^{c},
$$

where $e$ is an arbitrary constant, the gauge coupling constant. The problem, simply stated, is to solve the self duality equations:

$$
F_{\mu \nu}^{a}=+1 / 2 \varepsilon_{\mu \nu \lambda \varrho} F_{\lambda \varrho}^{a}
$$

(our convention is $\varepsilon_{1234} \equiv+1$ ) for the gauge potentials $A_{\mu}^{a}$ subject to the following requirements : 
(i) In all gauges, $A_{\mu}^{a}$ are static (independent of $x_{4}$ ): $\partial_{4} A_{\mu}^{a}=0$. In this case $A_{4}^{a}$ is referred to as the Higgs field.

(ii) In some gauge, $A_{\mu}^{a}$ are all real non-singular functions of $\left(x_{1}, x_{2}, x_{3}\right)$.

(iii) The gauge invariant quantity $H^{2} \equiv A_{4}^{a} A_{4}^{a}$ has the following asymptotic form :

$$
H^{2}=f^{2}-\frac{2 f n}{e r}+O\left(r^{-2}\right) \text { as } \quad r \rightarrow \infty,
$$

where $r^{2} \equiv x_{1}^{2}+x_{2}^{2}+x_{3}^{2}, f$ is an arbitrary constant with dimensions of inverse length and $n$ is a positive integer called the topological charge. We assume that ef $>0$. (To compare our formulas with Ward's one should set $f=1, e=2$.) The energy $E$ of the monopole is then:

$$
E \equiv \int 1 / 4 F_{\mu \nu}^{a} F_{\mu \nu}^{a} d^{3} x=1 / 2 \int \nabla^{2} H^{2} d^{3} x=4 \pi f g,
$$

where we have defined the magnetic charge $g$ to be $(n / e)$.

We now proceed to outline the general framework [2] within which we look for monopole solutions. We then present case by case the known $n=1$ and $n=2$ solutions and a new $n=3$ solution, after which an obvious pattern emerges allowing us to present the solution for arbitrary $n$.

\section{Formulations of the Self Duality Equations [2]}

We begin by defining the $2 \times 2$ matrix valued fields:

$$
A_{\mu} \equiv e \frac{\sigma^{a}}{2 i} A_{\mu}^{a} \quad \text { and } \quad F_{\mu \nu} \equiv e \frac{\sigma^{a}}{2 i} F_{\mu \nu}^{a}=\partial_{\mu} A_{\nu}-\partial_{\nu} A_{\mu}+\left[A_{\mu}, A_{\nu}\right]
$$

where $\sigma^{a}$ are the Pauli matrices. For real gauge fields, $A_{\mu}$ and $F_{\mu \nu}$ are antihermitian traceless matrices. We now analytically continue $A_{\mu}$ into complex space where $x_{1}, x_{2}, x_{3}, x_{4}$ are complex. The self duality equations (1.2) are then valid also in complex space, in a region containing real space where $x$ 's are real. Now consider the four new complex variables $p, \bar{p}, q$, and $\bar{q}$ defined by

$$
\sqrt{2} p \equiv x_{1}+i x_{2}, \quad \sqrt{2} \bar{p} \equiv x_{1}-i x_{2}, \quad \sqrt{2} q \equiv x_{3}-i x_{4}, \quad \sqrt{2} \bar{q} \equiv x_{3}+i x_{4} .
$$

The self duality equations (1.2) then reduce to:

$$
F_{p q}=0, \quad F_{\bar{p} \bar{q}}=0 \text { and } \quad F_{p \bar{p}}+F_{q \bar{q}}=0 .
$$

The equations $F_{p q}=0$ and $F_{\bar{p} \bar{q}}=0$ can be immediately integrated, since they are pure gauge, to give:

$$
A_{p}=D^{-1} D_{p}, \quad A_{q}=D^{-1} D_{q}, \quad A_{\bar{p}}=\bar{D}^{-1} \bar{D}_{\bar{p}}, \quad A_{\bar{q}}=\bar{D}^{-1} \bar{D}_{\bar{q}},
$$

where $D$ and $\bar{D}$ are arbitrary $2 \times 2$ complex matrix functions of $p, \bar{p}, q$, and $\bar{q}$ with determinant $=1$ and $D_{p} \equiv \partial_{p} D$, etc.

Gauge transformations are the transformations:

$$
D \rightarrow \bar{V}(\bar{p}, \bar{q}) D \mathscr{L}, \quad \bar{D} \rightarrow V^{-1}(p, q) \bar{D} \mathscr{L},
$$


where $\mathscr{L}$ is an arbitrary complex matrix function of $p, \bar{p}, q$, and $\bar{q}$ whereas $\bar{V}(V)$ is an arbitrary complex matrix function of $\bar{p}, \bar{q}(p, q)$ and we take the determinants of $\mathscr{L}, V$, and $\bar{V}$ to be one. Under the gauge transformation (2.5) the gauge potential $A_{\mu}$ and gauge field strength $F_{\mu \nu}$ transform as

$$
A_{\mu} \rightarrow \mathscr{L}^{-1} A_{\mu} \mathscr{L}+\mathscr{L}^{-1} \partial_{\mu} \mathscr{L}, \quad F_{\mu \nu} \rightarrow \mathscr{L}^{-1} F_{\mu \nu} \mathscr{L} .
$$

The energy density $F_{\mu \nu}^{a} F_{\mu \nu}^{a}=-\frac{2}{e^{2}} \operatorname{Tr}\left(F_{\mu \nu} F_{\mu \nu}\right)$ is invariant under gauge transformations. Let us define a matrix $J$ by:

$$
J \equiv D \bar{D}^{-1},
$$

then the remaining self duality equation $F_{p \bar{p}}+F_{q \bar{q}}=0$ becomes:

$$
\left(J^{-1} J_{p}\right)_{\bar{p}}+\left(J^{-1} J_{q}\right)_{\bar{q}}=0 .
$$

Under the gauge transformations (2.5), $J$ transforms as:

$$
J \rightarrow \bar{V}(\bar{p}, \bar{q}) J V(p, q) .
$$

Since $J$ is an arbitrary complex $2 \times 2$ matrix function with determinant one it can be parameterized as:

$$
J=\left[\begin{array}{cc}
\frac{1}{\phi} & \frac{\bar{\varrho}}{\phi} \\
\frac{\varrho}{\phi} & \frac{\phi^{2}+\varrho \varrho}{\phi}
\end{array}\right]
$$

where $\phi, \varrho$, and $\varrho$ are arbitrary and independent complex functions of $p, \bar{p}, q$, and $\bar{q}$. The self duality equations (2.8) in terms of $\phi, \varrho$, and $\varrho$ become:

$$
\begin{gathered}
\left(\partial_{p} \partial_{\bar{p}}+\partial_{q} \partial_{\bar{q}}\right) \ln \phi+\frac{\left(\varrho_{p} \bar{\varrho}_{p}+\varrho_{q} \bar{\varrho}_{\bar{q}}\right)}{\phi^{2}}=0, \\
\left(\frac{\varrho_{p}}{\phi^{2}}\right)_{\bar{p}}+\left(\frac{\varrho_{q}}{\phi^{2}}\right)_{\bar{q}}=0, \quad\left(\frac{\bar{\varrho}_{\bar{p}}}{\phi^{2}}\right)_{p}+\left(\frac{\bar{\varrho}_{\bar{q}}}{\phi^{2}}\right)_{q}=0 .
\end{gathered}
$$

To construct the gauge potentials $A_{\mu}$ from $J$ requires a selection of gauge [i.e., there are an infinite number of ways of factoring (2.10) in the form (2.7)]. We will work exclusively in Yang's $R$ gauge which is defined by $J \equiv R \bar{R}^{-1}$ where:

$$
R=\left(\begin{array}{cc}
\frac{1}{\sqrt{\phi}} & 0 \\
\frac{\varrho}{\sqrt{\phi}} & \sqrt{\phi}
\end{array}\right), \quad \bar{R}=\left(\begin{array}{cc}
\sqrt{\phi} & -\frac{\varrho}{\sqrt{\phi}} \\
0 & \frac{1}{\sqrt{\phi}}
\end{array}\right) .
$$

The gauge potentials in the $R$ gauge take the form:

$$
A_{u}=\left(\begin{array}{cc}
-\frac{\phi_{u}}{2 \phi} & 0 \\
\frac{\varrho_{u}}{\phi} & +\frac{\phi_{u}}{2 \phi}
\end{array}\right), \quad A_{\bar{u}}=\left(\begin{array}{cc}
\frac{\phi_{\bar{u}}}{2 \phi} & -\frac{\bar{\varrho}_{\bar{u}}}{\phi} \\
0 & -\frac{\phi_{\bar{u}}}{2 \phi}
\end{array}\right),
$$


where $u \equiv p, q$. Now because of requirement (i) the gauge potentials (2.13) must be $x_{4}$ independent and we require this to be true in all gauges which implies that the gauge transformation matrix $\mathscr{L}$ must be $x_{4}$ independent.

$$
\partial_{4} \mathscr{L}=0
$$

by virtue of (2.6). In particular $H^{2} \equiv A_{4}^{a} A_{4}^{a}=-\frac{2}{e^{2}} \operatorname{Tr}\left(A_{4} A_{4}\right)$ is gauge invariant.

Even if the gauge potentials $A_{\mu}$ derived from (2.13) [e.g., $\left.A_{4}=\frac{-i}{\sqrt{2}}\left(A_{q}-A_{q}\right)\right]$ are static, they will not in general be antihermitian as demanded by requirement (ii). For real gauge fields $A_{\mu}^{+} \doteq-A_{\mu}$ (the symbol $\doteq$ is used for equations valid only for real values of $\left.x_{1}, x_{2}, x_{3}, x_{4}\right)$, we have from (2.4) and (2.7)

$$
A_{\mu}^{+} \doteq-A_{\mu} \Rightarrow \bar{D} \doteq\left(D^{+}\right)^{-1} \Rightarrow J=D \bar{D}^{-1} \doteq D D^{+}
$$

i.e., for real gauge fields $J$ is a positive definite $2 \times 2$ hermitian matrix. A necessary and sufficient condition to meet requirement (ii) ${ }^{1}$ is that we be able to find matrices $\bar{V}(\bar{p}, \bar{q})$ and $V(p, q)$ in Eq. (2.9) such that $\bar{V}(\bar{p}, \bar{q}) R \bar{R}^{-1} V(p, q)$ is a positive definite hermitian matrix. If we can find such matrices $V$ and $\bar{V}$, then the gauge transformation matrix $\mathscr{L}$ in (2.6) is simply a square root of the matrix:

$$
\mathscr{L} \mathscr{L}^{+} \doteq\left(R^{+} \bar{V}^{+} V^{-1} \bar{R}\right)^{-1}
$$

and this matrix $\mathscr{L}$ will make $A_{\mu}$ and $F_{\mu \nu}$ antihermitian as demanded by requirement (ii). We now proceed to the solution of the self duality equations (2.11) and the implementation of requirements (i)-(iii).

\section{Implementation of Requirement (i)}

We will begin by insuring that the gauge potentials (2.13) are $x_{4}$ independent. We do this by looking for solutions of the self duality equations (2.11) in the following form:

$$
\phi \equiv e^{i e f x_{4}} Q_{\phi}, \quad \varrho \equiv e^{i e f x_{4}} Q_{\varrho}, \quad \bar{\varrho} \equiv e^{i e f x_{4}} Q_{\bar{\varrho}},
$$

where

$$
Q_{\phi}, Q_{\varrho}, Q_{\bar{\varrho}} \text { are functions of } x_{1}, x_{2}, x_{3} \text { only. }
$$

The gauge potentials (2.13) constructed from (3.1) are manifestly $x_{4}$ independent.

\section{Solution of the Sefl Duality Equations (2.11): The Atiyah-Ward Ansatz [3]}

The Atiyah-Ward ansatz can be stated as follows. Let $\left({ }_{n} \phi,{ }_{n} \varrho,{ }_{n} \varrho\right)$ be solutions to the self duality equations $(2.11)$, then so are $\left({ }_{n+1} \phi,{ }_{n+1} \varrho,{ }_{n+1} \varrho \bar{\varrho}\right)$ where :

$$
{ }_{n+1} \phi=\frac{{ }_{n} \phi^{2}+{ }_{n} \varrho_{n} \bar{\varrho}}{{ }_{n} \phi}
$$

1 We must, of course, also check that the gauge fields are nonsingular functions of $x_{1}, x_{2}$, and $x_{3}$ 


$$
\begin{aligned}
& { }_{n+1} \varrho_{p}=-\left[\frac{{ }_{n} \phi^{2}+{ }_{n} \varrho_{n} \varrho}{{ }_{n} \phi}\right]^{2}\left[\frac{{ }_{n} \varrho}{{ }_{n}^{2} \phi^{2}+{ }_{n} \varrho_{n} \varrho}\right]_{\bar{q}} ; \quad{ }_{n+1} \varrho_{q}=+\left[\frac{{ }_{n} \phi^{2}+{ }_{n} \varrho_{n} \varrho}{{ }_{n} \phi}\right]^{2}\left[\frac{{ }_{n} \varrho}{\phi^{2}+{ }_{n} \varrho_{n} \varrho}\right]_{\bar{p}}, \\
& { }_{n+1} \bar{\varrho}_{\bar{p}}=+\left[\frac{{ }_{n} \phi^{2}+{ }_{n} \varrho_{n} \bar{\varrho}}{{ }_{n} \phi}\right]^{2}\left[\frac{{ }_{n} \bar{\varrho}}{{ }_{n}^{2}+{ }_{n} \varrho_{n} \bar{\varrho}}\right]_{q} ; \quad n+1 \bar{\varrho}_{\bar{q}}=-\left[\frac{{ }_{n} \phi^{2}+{ }_{n} \varrho_{n} \bar{\varrho}}{{ }_{n} \phi}\right]^{2}\left[\frac{{ }_{n} \bar{\varrho}}{{ }_{n} \phi^{2}+{ }_{n} \varrho_{n} \bar{\varrho} \varrho}\right]_{p} \text {. }
\end{aligned}
$$

The proof of (4.1) is given in Appendix A. Thus the Atiyah-Ward ansatz provides a recursive method of obtaining solutions to (2.11). Let us now define:

$$
(\phi, \varrho, \varrho) \text { associated with the Atiyah-Ward ansatz } \mathscr{A}_{n} \equiv\left({ }_{n} \phi,{ }_{n} \varrho,{ }_{n} \varrho\right) \text {. }
$$

The recursion starts with the $\mathscr{A}_{1}$ ansatz which is defined by:

$$
\mathscr{A}_{1} \text { ansatz: }{ }_{1} \varrho_{p}={ }_{1} \phi_{\bar{q}}, \quad{ }_{1} \varrho_{q}=-{ }_{1} \phi_{\bar{p}}, \quad{ }_{1} \bar{\varrho}_{\bar{p}}={ }_{1} \phi_{q}, \quad{ }_{1} \bar{\varrho}_{\bar{q}}=-{ }_{1} \phi_{p},
$$

which implies:

$$
{ }_{1} \phi_{p \bar{p}}+{ }_{1} \phi_{q \bar{q}}=0 .
$$

We look for solutions of (4.4) in the form (3.1):

$$
{ }_{1} \phi=e^{i e f x_{4}} \Lambda_{0},
$$

which implies a Helmholtz equation for $\Lambda_{0}$

$$
\nabla^{2} \Lambda_{0}=(e f)^{2} \Lambda_{0} .
$$

We will take the general solution of (4.6) to be:

$$
\begin{aligned}
\Lambda_{0} & =\sum_{k=1}^{n} \alpha_{k} \frac{\sinh \left(e f r_{k}\right)}{r_{k}}, \\
r_{k}^{2} & \equiv x_{1}^{2}+x_{2}^{2}+\left(x_{3}-z_{k}\right)^{2},
\end{aligned}
$$

where $\alpha_{k}$ and $z_{k}$ are arbitrary complex constants constrained only by the requirement that $\Lambda_{0}$ be a real function of $x_{1}, x_{2}$, and $x_{3}$. If we now define a real function $\Lambda_{1}$ by

$$
\Lambda_{0} \equiv \bar{p}^{-1} \partial_{p} \Lambda_{1} \equiv p^{-1} \hat{o}_{\bar{p}} \Lambda_{1}
$$

then we can integrate Eq. (4.3) to give

$$
\begin{gathered}
{ }_{1} \phi=e^{i e f x_{4}} \Lambda_{0}, \quad{ }_{1} \varrho=e^{i e f x_{4}}(\sqrt{2} \bar{p})^{-1}\left(\partial_{3}+e f\right) \Lambda_{1} \\
{ }_{1} \bar{\varrho}=e^{i e f x_{4}}(\sqrt{2} p)^{-1}\left(\partial_{3}-e f\right) \Lambda_{1} .
\end{gathered}
$$

Note that when we integrate $\Lambda_{0}$ to obtain $\Lambda_{1}$ we do not keep any constants of integration.

\section{Integration of the $\mathscr{A}_{n}$ Ansatz}

In Eq. (4.9) we have completely integrated the $\mathscr{A}_{1}$ ansatz. Corrigan et al. [4] have integrated Eqs. (4.1) for any $n \geqq 2$. Their solution begins by defining $(2 n+1)$ functions $\Delta_{\ell}$, where $-n \leqq \ell \leqq n$, which satisfy the following equations:

$$
\partial_{p} \Delta_{\ell}=-\partial_{\bar{q}} \Delta_{\ell+1}, \quad \partial_{q} \Delta_{\ell}=\partial_{\bar{p}} \Delta_{\ell+1} .
$$


Then the $(\phi, \varrho, \bar{\varrho})$ associated with the $\mathscr{A}_{n}$ ansatz for $n \geqq 2$ are given by:

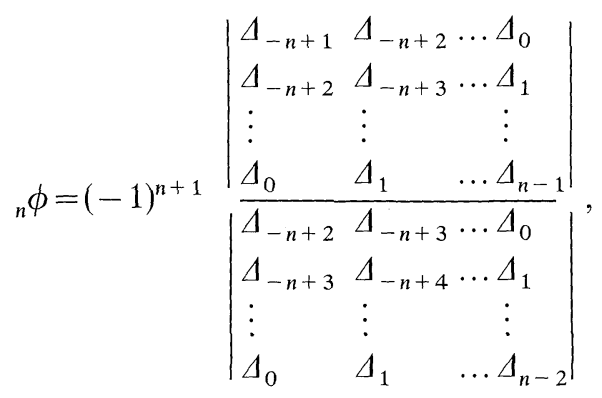

$$
\begin{aligned}
& { }_{n} Q=-\frac{\left|\begin{array}{ccc}
\Delta_{-n} & \Delta_{-n+1} & \ldots \Delta_{-1} \\
\Delta_{-n+1} & \Delta_{-n+2} & \ldots \Delta_{0} \\
\vdots & \vdots & \vdots \\
\Delta_{-1} & \Delta_{0} & \ldots \Delta_{n-2}
\end{array}\right|}{\left|\begin{array}{ccc}
\Delta_{-n+2} & \Delta_{-n+3} & \ldots \Delta_{0} \\
\Delta_{-n+3} & \Delta_{-n+4} & \ldots \Delta_{1} \\
\vdots & \vdots & \vdots \\
\Delta_{0} & \Delta_{1} & \ldots \Delta_{n-2}
\end{array}\right|}
\end{aligned}
$$

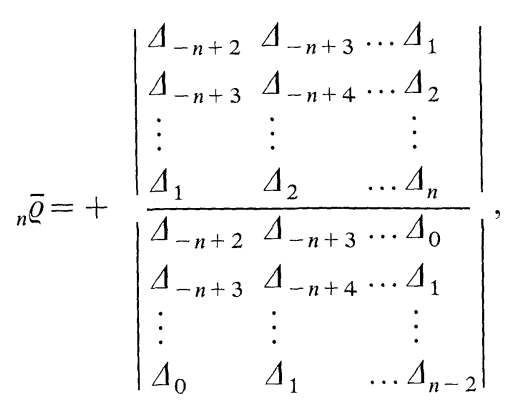

Where $|\ldots|$ designates determinant. Equations (4.1a) and (5.2a) give the following useful relation:

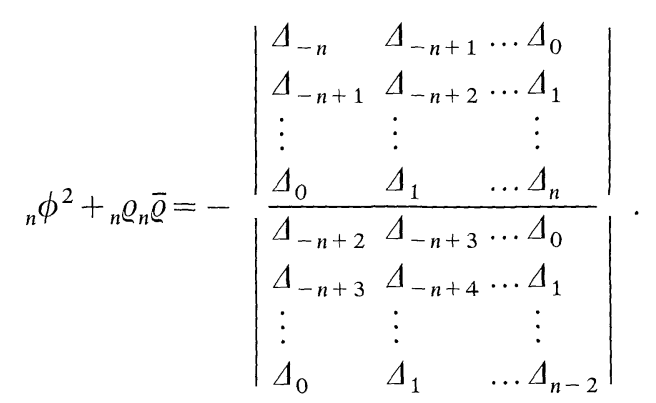

Let us define the real functions $\Lambda_{n}$ for $n \geqq 2$ by:

$$
\Lambda_{n} \equiv \bar{p}^{-1} \partial_{p} \Lambda_{n+1} \equiv p^{-1} \partial_{\bar{p}} \Lambda_{n+1},
$$


then it follows by induction that:

$$
\begin{aligned}
\Delta_{0} & ={ }_{1} \phi=e^{i e f x_{4}} \Lambda_{0}, \\
\Delta_{-n} & =(-1)^{n} e^{i e f x_{4}}(\sqrt{2} \bar{p})^{-n}\left(\partial_{3}+e f\right)^{n} \Lambda_{n}, \\
\Delta_{+n} & =e^{i e f x_{4}}(\sqrt{2} p)^{-n}\left(\partial_{3}-e f\right)^{n} \Lambda_{n} .
\end{aligned}
$$

Note that when we integrate $\Lambda_{n}$ to obtain $\Lambda_{n+1}$ we do not keep any constants of integration. The $\Lambda$ 's are given by:

$$
\begin{aligned}
& \Lambda_{0}=(e f) \sum_{k=1}^{n} \alpha_{k} \frac{\sinh \left(e f r_{k}\right)}{e f r_{k}} \\
& \Lambda_{1}=(e f)^{-1} \sum_{k=1}^{n} \alpha_{k} \cosh \left(e f r_{k}\right) \\
& \Lambda_{2}=(e f)^{-3} \sum_{k=1}^{n} \alpha_{k}\left(e f r_{k}\right) \sinh \left(e f r_{k}\right)-(e f)^{-2} \Lambda_{1} \\
& \Lambda_{3}=(e f)^{-5} \sum_{k=1}^{n} \alpha_{k}\left(e f r_{k}\right)^{2} \cosh \left(e f r_{k}\right)-3(e f)^{-2} \Lambda_{2} \\
& \Lambda_{4}=(e f)^{-7} \sum_{k=1}^{n} \alpha_{k}\left(e f r_{k}\right)^{3} \sinh \left(e f r_{k}\right)-6(e f)^{-2} \Lambda_{3}-3(e f)^{-4} \Lambda_{2}
\end{aligned}
$$

\section{Implementation of Requirement (iii):}

\section{A Remarkable Superposition Formula [5]}

It is proven in Appendix B that for the $\mathscr{A}_{n}$ ansatz the gauge invariant quantity $H^{2}=A_{4}^{a} A_{4}^{a}$ is given by:

$$
\mathscr{A}_{n} \text { ansatz: } H^{2}=f^{2}+\frac{1}{e^{2}} \sum_{k=1}^{n}\left[-\nabla^{2} \ln _{k} \phi\right] \text {, }
$$

which represents a remarkable superposition formula for the energy density of the monopole solutions. Now from (4.7) and (4.9) we see that $\ln _{1} \phi \rightarrow e f\left(r+i x_{4}\right)+O(\ln r)$ as $r \rightarrow \infty$. The Atiyah-Ward transformations do not change the asymptotic behavior of ${ }_{1} \phi$ so that

$$
\ln _{1} \phi \rightarrow e f\left(r+i x_{4}\right)+O(\ln r) \Rightarrow \ln _{n} \phi \rightarrow e f\left(r+i x_{4}\right)+O(\ln r) \text { as } r \rightarrow \infty .
$$

Substituting (6.2) into (6.1) we see that the asymptotic behavior of $H^{2}$ is

$$
\mathscr{A}_{n} \text { ansatz: } H^{2} \rightarrow f^{2}-\frac{2 f n}{e r}+O\left(r^{-2}\right) \text { as } r \rightarrow \infty,
$$

which is precisely what requirement (iii) demands. Thus the $n^{\text {th }}$ Atiyah-Ward ansatz $\mathscr{A}_{n}$ is guaranteed to give topological charge $n$ provided only $\ln _{1} \phi \rightarrow e f\left(r+i x_{4}\right)+O(\ln r)$ as $r \rightarrow \infty$. 


\section{Implementation of Requirement (ii)}

We have already shown that reality of the gauge field is assured if we can find matrices $\bar{V}(\bar{p} \bar{q})$ and $V(p q)$ such that $\bar{V} R \bar{R}^{-1} V$ is a positive definite hermitian matrix. Equations (2.13) and (6.1) shows that to assure the gauge fields are nonsingular all we have to verify that ${ }_{n} \phi$ never vanishes for each $n$.

We now proceed directly to study how the above formalism applies to the $n=1,2,3$ monopole solutions, after which we present the arbitrary $n$ monopole solution.

\section{The $n=1$ Monopole Solution [6]}

Here we take the $\mathscr{A}_{1}$ ansatz with

$$
\Lambda_{0}=\frac{\sinh (e f r)}{r} .
$$

The matrices $\bar{V}(\bar{p}, \bar{q})$ and $V(p, q)$ which make $\bar{V} R \bar{R}^{-1} V$ a positive definite hermitian matrix for $\left({ }_{1} \phi,{ }_{1} \varrho,{ }_{1} \varrho\right)$ constructed from $(8.1)$ are:

$$
\bar{V}(\bar{p}, \bar{q})=I=\left(\begin{array}{ll}
1 & 0 \\
0 & 1
\end{array}\right), \quad V(p, q)=\left(\begin{array}{cc}
0 & (\sqrt{2} p)^{-1} \\
-\sqrt{2} p & 0
\end{array}\right)
$$

and the gauge transformation matrix $\mathscr{L}$ is formed by:

$$
\mathscr{L} \mathscr{L}^{+}=\left(R^{+} \bar{V}^{+} V^{-1} \bar{R}\right)^{-1}=e^{-e f(\sigma \cdot \mathbf{x})} \Rightarrow \mathscr{L}=e^{-1 / 2 e f(\sigma \cdot \mathbf{x})} .
$$

Furthermore (8.1) never vanishes so that we indeed have the original real nonsingular spherically symmetric $n=1$ monopole solution.

\section{Ward's $n=2$ Monopole Solution}

Here we take the $\mathscr{A}_{2}$ ansatz with:

$$
\Lambda_{0}=\frac{\sinh \left(e f r_{1}\right)}{r_{1}}+\frac{\sinh \left(e f r_{2}\right)}{r_{2}}
$$

where

$$
r_{1}^{2}=x_{1}^{2}+x_{2}^{2}+\left(x_{3}-z_{0}\right)^{2}, \quad r_{2}^{2}=x_{1}^{2}+x_{2}^{2}+\left(x_{3}+z_{0}\right)^{2} .
$$

Guided by Eq. (8.2), we now choose the matrices $\bar{V}(\bar{p} \bar{q})$ and $V(p q)$ to be:

$$
\bar{V}(\bar{p} \bar{q})=I=\left(\begin{array}{ll}
1 & 0 \\
0 & 1
\end{array}\right), \quad V(p, q)=\left(\begin{array}{cc}
0 & \gamma^{-1}(\sqrt{2} p)^{-2} \\
-\gamma(\sqrt{2} p)^{2} & 0
\end{array}\right)
$$

and require that $\bar{V} R \bar{R}^{-1} V$ be a positive definite hermitian matrix for $\left({ }_{2} \phi,{ }_{2} \varrho,{ }_{2} \varrho\right)$ constructed from (9.1). After some tedious algebra one finds in order to have real gauge fields :

$\gamma$ must be a real constant,

$$
-\gamma^{2}(2 p \vec{p})^{2} e^{-2 i e f x_{4}}\left({ }_{2} \phi^{2}+{ }_{2} \varrho_{2} \bar{\varrho}\right)=-\left(4 \gamma z_{0}\right)^{2}=+1 \text {. }
$$


Note that in calculating (9.4b) we have made essential use of Eq. (5.3). Equations (9.4a) and (9.4b) imply:

$$
z_{0}=+i c, \quad c \text { is a real constant }, \quad \gamma=(4 c)^{-1} .
$$

It remains to check that the gauge fields are nonsingular and we find that (again by tedious algebra)

$$
{ }_{1} \phi_{2} \phi \neq 0 \Rightarrow c=\frac{\pi}{2 e f}
$$

so that there are no free parameters left in the solution. Note that in Eq. (9.6) we always calculate ${ }_{1} \phi$ and ${ }_{2} \phi$ using $\Lambda_{0}$ given by Eq. (9.1).

\section{The New $n=3$ Monopole Solution}

Here we take the $\mathscr{A}_{3}$ ansatz with

$$
\Lambda_{0}=\frac{\sinh \left(e f r_{1}\right)}{r_{1}}+\frac{\sinh \left(e f r_{2}\right)}{r_{2}}+2 \frac{\sinh (e f r)}{r},
$$

where

$$
r_{1}^{2}=x_{1}^{2}+x_{2}^{2}+\left(x_{3}-z_{0}\right)^{2} ; \quad r_{2}^{2}=x_{1}^{2}+x_{2}^{2}+\left(x_{3}+z_{0}\right)^{2} ; \quad r^{2}=x_{1}^{2}+x_{2}^{2}+x_{3}^{2} .
$$

Guided by Eqs. (8.2) and (9.3), we now choose the matrices $\bar{V}(\bar{p} \bar{q})$ and $V(p q)$ to be:

$$
\bar{V}(\bar{p} \bar{q})=I=\left(\begin{array}{ll}
1 & 0 \\
0 & 1
\end{array}\right), \quad V(p, q)=\left(\begin{array}{cc}
0 & \gamma^{-1}(\sqrt{2} p)^{-3} \\
-\gamma(\sqrt{2} p)^{3} & 0
\end{array}\right)
$$

and require that $\bar{V} R \bar{R}^{-1} V$ be a positive hermitian matrix for $\left({ }_{3} \phi,{ }_{3} \varrho,{ }_{3} \varrho\right)$ constructed from (10.1). The calculations are now so complicated that we had to resort to the symbol manipulation computer program MACSYMA, and we find that in order to have real gauge fields:

$$
\begin{gathered}
\gamma \text { must be a real constant, } \\
-\gamma^{2}(2 p \bar{p})^{3}\left({ }_{3} \phi^{2}+{ }_{3} \varrho_{3} \varrho\right) e^{-2 i e f x_{4}}=\left(8 \gamma z_{0}^{2}\right)^{2}=+1 .
\end{gathered}
$$

Note that in calculating (10.4b) we have again made essential use of Eq. (5.3).

It remains to check that the gauge fields are nonsingular and one fiends, using MACSYMA :

$$
{ }_{1} \phi_{2} \phi_{3} \phi \neq 0 \Rightarrow z_{0}=\frac{i \pi}{e f}
$$

so that again there are no free parameters left in the solution. Note that in Eq. (10.5) we always calculate ${ }_{1} \phi,{ }_{2} \phi$, and ${ }_{3} \phi$ using $\Lambda_{0}$ given by Eq. (10.1). 


\section{The Arbitrary n-Monopole Solution}

The $n=1,2,3$ monopole solutions follow an obvious pattern which has allowed us to guess and indeed verify the following central result of this paper. To obtain a monopole solution of arbitrary topological charge $n \geqq 1$ one should take the $\mathscr{A}_{n}$ ansatz with

$$
\Lambda_{0}=\sum_{k=1}^{n} \alpha_{k} \frac{\sinh \left(e f r_{k}\right)}{r_{k}}
$$

where

$$
\begin{aligned}
& r_{k}^{2}=x_{1}^{2}+x_{2}^{2}+\left(x_{3}-z_{k}\right)^{2}, \\
& z_{k}=\left[\frac{(n+1)}{2}-k\right]\left(\frac{\pi i}{e f}\right), \\
& \alpha_{k}=\frac{(n-1) !}{(k-1) !(n-k) !} .
\end{aligned}
$$

Note that $z_{k}-z_{k-1}=i \pi /(e f)$ and $\alpha_{k}$ are the binomial coefficients.

To verify the above result we choose the matrices $\bar{V}(\bar{p} \bar{q})$ and $V(p q)$ to be:

$$
\bar{V}(\bar{p} \bar{q})=I=\left(\begin{array}{ll}
1 & 0 \\
0 & 1
\end{array}\right), \quad V(p, q)=\left(\begin{array}{cc}
0 & \gamma^{-1}(\sqrt{2} p)^{-n} \\
-\gamma(\sqrt{2} p)^{n} & 0
\end{array}\right),
$$

where $\gamma$ is a real constant, and require that $\bar{V} R \bar{R}^{-1} V$ be a positive definite hermitian matrix for $\left({ }_{n} \phi,{ }_{n} \varrho, \varrho\right)$ constructed from (11.1). We must also check that ${ }_{1} \phi_{2} \phi \ldots{ }_{n} \phi$ never vanishes. From (5.2a) one finds :

$$
{ }_{1} \phi_{2} \phi \ldots{ }_{n} \phi=(-1)^{\frac{n(n+3)}{2}}\left|\begin{array}{llll}
\Delta_{-n+1} & \Delta_{-n+2} & \ldots & \Delta_{0} \\
\Delta_{-n+2} & \Delta_{-n+3} & \ldots & \Delta_{1} \\
\vdots & \vdots & \vdots \\
\Delta_{0} & \Delta_{1} & \ldots & \Delta_{n-1}
\end{array}\right| .
$$

The key to verifying our result is to study what happens on the $x_{3}$ axis where $x_{1}=x_{2}=0$. Equations (11.3) and (5.5) imply that if $H^{2}$ as defined in Eq. (6.1) is not to develop line singularities on the $x_{3}$ axis one must have:

$$
(2 p \bar{p})^{\ell} \Delta_{-\ell} \Delta_{+\ell}=0, \quad \text { for } 1 \leqq \ell \leqq n, \quad \text { and } \quad x_{1}=x_{2}=0 .
$$

By virtue of Eqs. (5.5b) and (5.5c) we choose to satisfy (11.4) by requiring:

$$
\Lambda_{\ell}=0 \text { for } 1 \leqq \ell<n \text { and } x_{1}=x_{2}=0 .
$$

Of course $\Lambda_{0}$ must never vanish. It is tedious but straightforward to verify that (11.1) satisfies (11.5). In order to have real gauge fields on the $x_{3}$ axis one finds, using Eqs. (5.3) and (11.4), that :

$$
\begin{aligned}
-\gamma^{2}(2 p \bar{p})^{n} e^{-2 i e f x_{4}}\left({ }_{n} \phi^{2}+{ }_{n} \varrho_{n} \bar{\varrho}\right) & =\gamma^{2}(2 p \bar{p})^{n} e^{-2 i e f x_{4}} \Delta_{-n} \Delta_{+n} \\
& =\left[\gamma\left(\frac{2 \pi}{e f}\right)^{n-1}(n-1) !\right]^{2}=+1
\end{aligned}
$$


Thus requiring the gauge fields be real and nonsingular on the $x_{3}$ axis uniquely fixes the solution to be (11.1). One can prove reality of the gauge fields over all three dimensional Euclidean space, but this is much more difficult and is presented in [9]. In [9] we have verified regularity of the solutions in various regions, but we have not yet shown that these regions cover the three dimensional Euclidean space.

\section{Discussions}

The axially symmetric monopoles presented in this paper contain no free parameters. However, solutions containing free parameters are known to exist [7] and presumably they are not axially symmetric. It appears likely that the AtiyahWard ansatz may still provide the framework to study such nonaxially symmetric monopoles.

Explicit forms of the gauge potentials in various real gauges can be found in [2] and a real gauge in which mirror symmetry is manifest can be found in [8].

\section{Appendix A: Proof of Equation (4.1) [5]}

The proof of Eq. (4.1) rests on two theorems which we now state:

Theorem 1. If $(\phi, \varrho, \bar{\varrho})$ satisfy Eq. (2.11), then so do $\left(\phi^{I}, \varrho^{I}, \varrho^{I}\right)$ defined by

$$
\phi^{I}=\frac{\phi}{\phi^{2}+\varrho \varrho}, \quad \varrho^{I}=\frac{\bar{\varrho}}{\phi^{2}+\varrho \bar{\varrho}}, \quad \bar{\varrho}^{I}=\frac{\varrho}{\phi^{2}+\varrho \varrho}
$$

and furthermore the gauge potentials derived from $\left(\phi^{I}, \varrho^{I}, \bar{\varrho}^{I}\right)$ are gauge transformations of those derived from $(\phi, \varrho, \varrho)$.

Proof.

$$
J^{I}=\left(\begin{array}{cc}
\frac{1}{\phi^{I}} & \frac{\bar{\varrho}^{I}}{\phi^{I}} \\
\frac{\varrho^{I}}{\phi^{I}} & \frac{\phi^{I^{2}}+\varrho^{I} \bar{\varrho}^{I}}{\phi^{I}}
\end{array}\right)=\left(\begin{array}{cc}
\frac{\phi^{2}+\varrho \bar{\varrho}}{\phi} & \frac{\varrho}{\phi} \\
\frac{\bar{\varrho}}{\phi} & \frac{1}{\phi}
\end{array}\right)=\left(\begin{array}{cc}
0 & -i \\
-i & 0
\end{array}\right) J\left(\begin{array}{ll}
0 & i \\
i & 0
\end{array}\right)
$$

so that in (2.9) we have $V=\left(\begin{array}{ll}0 & i \\ i & 0\end{array}\right)$ and $\bar{V}=\left(\begin{array}{cc}0 & -i \\ -i & 0\end{array}\right)$.

Theorem 2. If $(\phi, \varrho, \bar{\varrho})$ satisfy Eq. (2.11), then so do $\left(\phi^{B}, \varrho^{B}, \varrho^{-B}\right)$ defined by:

$$
\phi^{B}=\frac{1}{\phi} ; \quad \varrho_{p}^{B}=-\frac{\bar{\varrho}_{\bar{q}}}{\phi^{2}} ; \quad \varrho_{q}^{B}=\frac{\bar{\varrho}_{\bar{p}}}{\phi^{2}} ; \quad \bar{\varrho}_{\bar{p}}^{B}=\frac{\varrho_{q}}{\phi^{2}} ; \quad \bar{\varrho}_{\bar{q}}^{B}=-\frac{\varrho_{p}}{\phi^{2}} .
$$

The proof of Theorem 2 follows from the assumption that $\varrho$ is integrable so that $\varrho_{p q}=\varrho_{q p}$, etc.

Note that $I$ is a discrete transformation, since when operated twice it gives an identity (i.e., $\phi^{I I}=\phi, \varrho^{I I}=\varrho, \ldots$ etc.). Furthermore, acting with the operator $B$ twice is a trivial operation (i.e., $\phi^{B B}=\phi, \varrho_{p}^{B B}=\varrho_{p}$, .. etc.) in that it does not change the 
gauge potentials. Therefore, in order to use $B$ more than once, one must interpose the $I$ operation of Theorem 1 between two $B$ 's.

The Atiyah-Ward ansatz $\mathscr{A}_{n}(n=1,2,3, \ldots)$ can now be defined by the following chain of operations

$$
\mathscr{A}_{1} \stackrel{(B I)}{\longrightarrow} \mathscr{A}_{2} \stackrel{(B I)}{\longrightarrow} \mathscr{A}_{3} \stackrel{(B I)}{\longrightarrow} \ldots
$$

where $\mathscr{A}_{1}$ is the first ansatz defined by Eq. (4.3) and $(B I)$ means operate with $I$ first and then with $B$. Thus we have proved Eq. (4.1).

For comparison purposes we note that the relation of what we call the AtiyahWard ansatz $\mathscr{A}_{n}$ to what Corrigan, Fairlie, Goddard, and Yates call ansatz $R_{\ell}$ is the following: $\mathscr{A}_{n} \equiv I R_{n+1}$. With this transcription the formulas of Sect. $\mathrm{V}$ immediately follow.

\section{Appendix B: Proof of Equation (6.1) [5]}

From Eq. (2.13) and the fact $A_{4}=\frac{-i}{\sqrt{2}}\left(A_{q}-A_{\bar{q}}\right)$ we have

$$
H^{2}=\frac{-2}{e^{2}} \operatorname{Tr}\left(A_{4} A_{4}\right)=\frac{1}{e^{2}}\left[\frac{\phi_{3}^{2}+2 \varrho_{q} \bar{\varrho}_{\bar{q}}}{\phi^{2}}\right] \text {. }
$$

Now in Appendix A we proved that $I$ is a gauge transformation so that $H^{2}$ which is gauge invariant is invariant under it

$$
H^{2}(I) \equiv \frac{-2}{e^{2}} \operatorname{Tr}\left(A_{4}^{I} A_{4}^{I}\right)=\frac{1}{e^{2}}\left[\frac{\phi_{3}^{I^{2}}+2 \varrho_{q}^{I} \bar{\varrho}_{\bar{q}}^{I}}{\phi^{I^{2}}}\right]=\frac{1}{e^{2}}\left[\frac{\phi_{3}^{2}+2 \varrho_{q} \bar{\varrho}_{\bar{q}}}{\phi^{2}}\right] .
$$

On the other hand, under the $B$ transformation:

$$
\begin{aligned}
H^{2}(B) & \equiv-\frac{2}{e^{2}} \operatorname{Tr}\left(A_{4}^{B} A_{4}^{B}\right)=\frac{1}{e^{2}}\left[\frac{\phi_{3}^{B^{2}}+2 \varrho_{q}^{B} \bar{\varrho}_{\bar{q}}^{B}}{\phi^{B^{2}}}\right]=\frac{1}{e^{2}}\left[\frac{\phi_{3}^{2}-2 \varrho_{p} \bar{\varrho}_{\bar{p}}}{\phi^{2}}\right] \\
& =\frac{1}{e^{2}}\left[\frac{\phi_{3}^{2}+2 \varrho_{q} \bar{\varrho}_{\bar{q}}}{\phi^{2}}\right]+\frac{1}{e^{2}} \nabla^{2} \ln \phi,
\end{aligned}
$$

where we have used (A.2) in (B.3) and (2.11a) and (3.1) in (B.4). Thus for the operation $(B I)$ :

$$
H^{2}(B I)=-\frac{2}{e^{2}} \operatorname{Tr}\left(A_{4}^{B I} A_{4}^{B I}\right)=-\frac{2}{e^{2}} \operatorname{Tr}\left(A_{4} A_{4}\right)-\frac{1}{e^{2}} \nabla^{2} \ln \phi^{B I} .
$$

For the $\mathscr{A}_{1}$ ansatz we have, using Eqs. (4.3), (2.11a), and (3.1):

$$
\begin{aligned}
H^{2}\left(\mathscr{A}_{1}\right) & =\frac{1}{e^{2}}\left[\frac{{ }_{1} \phi_{3}^{2}+2{ }_{1} \varrho_{q 1} \bar{\varrho}_{\bar{q}}}{{ }_{1} \phi^{2}}\right]=\frac{1}{e^{2}}\left[\frac{2{ }_{1} \varrho_{q 1} \bar{\varrho}_{\bar{q}}}{{ }_{1} \phi^{2}}+\frac{2_{1} \varrho_{p 1} \bar{\varrho}_{\bar{p}}}{{ }_{1} \phi^{2}}-\left(\partial_{4} \ln _{1} \phi\right)^{2}\right] \\
& =-\frac{1}{e^{2}} \nabla^{2} \ln _{1} \phi+f^{2} .
\end{aligned}
$$

Equation (6.1) follows by induction. 
Acknowledgements. I thank Dr. A. Sinha and Dr. Ling Lie Chau Wang for thoughtful discussions on the monopole problem. I am especially grateful to Dr. P. Rossi for pointing out to me the possibility that the pattern described in Sect. XI may exist and to Dan Meiron who set me up with the MACSYMA system, without which this paper would not have been possible.

\section{References}

1. Ward, R.S.: Commun. Math. Phys. 79, 317-325 (1981)

2. Prasad, M.K., Sinha, A., Wang, Ling Lie Chau: A systematic framework for generating multimonopole solutions. Phys. Rev. D (to be published)

3. Atiyah, M.F., Ward, R.S.: Commun. Math. Phys. 55, 117-124 (1977)

4. Corrigan, E.F., Fairlie, D.B., Yates, R.G., Goodard, P.: Commun. Math. Phys. 58, 223-240 (1978)

5. Prasad, M.K. : Physica 1D, 167-191 (1980); Phys. Rev. D 17, 2177-2178 (1978)

6. Prasad, M.K., Sommerfield, C.M. : Phys. Rev. Lett. 35, 760-762 (1975)

7. Jaffe, A., Taubes, C.: Vortices and monopoles. Boston: Birkhäuser 1980

8. Prasad, M.K., Rossi, P.: Mirror symmetry and exact multimonopole solutions. Phys. Rev. D 23, 1795-1799 (1981)

9. Prasad, M.K., Rossi, P.: Construction of exact multimonopole solutions. MIT-CTP preprint No. 903, December 1980

Communicated by A. Jaffe

Received October 28, 1980; in revised form March 11, 1981 
